\title{
Large Eddy Simulations of Nanoparticle Synthesis in Spray Flames
}

\author{
S.-J. Baik ${ }^{* 1}$, J. Sellmann ${ }^{1}$, A. M. Kempf ${ }^{1,2}$ \\ ${ }^{1}$ Chair of Fluid Dynamics, Institute for Combustion and Gasdynamics (IVG), University of \\ Duisburg-Essen, Duisburg, Germany \\ ${ }^{2}$ Center for Nano-Integration Duisburg-Essen (CENIDE), University of Duisburg-Essen, \\ Duisburg, Germany \\ ${ }^{*}$ Corresponding author email: seung.baik@uni-due.de
}

\begin{abstract}
The "SpraySyn" burner [1] has been developed to investigate nanoparticle synthesis from spray flames by experiment and simulation. The spray is surrounded by a premixed pilot flame to stabilize combustion. Since the precursors are dissolved in the liquid fuel (ethanol) and evaporized by interaction with the hot (pilot) flame products, an accurate comprehension of the spray flame structure is necessary to study nanoparticle synthesis. In the present work, the in-house large eddy simulations (LES) code PsiPhi is applied to the SpraySyn burner to investigate the characteristics of spray flames and nanoparticle synthesis. The ethanol liquid droplets are described by Lagrangian particles, and gas-phase combustion is modeled by the flamelet generated manifold (FGM) approach combined with the artificial flame thickening (ATF) technique. The Iron(III) oxide nanoparticle formation and growth are modeled in terms of number-, surface-, and volume-concentration by a monodisperse model.
\end{abstract}

\section{Keywords}

Large-eddy simulation, Nanoparticle synthesis, Spray combustion

\section{Introduction}

Nanoparticle synthesis from the gas phase is a popular route to produce powders with a large variety of composition and size properties. Since flames can provide stable thermal conditions for the formation of nanoparticles, many of the nanoparticles' production is based on flames. At laboratory-scale the synthesis processes are mostly laminar at moderate Reynolds number and low pressure. However, in pilot scale operation these laminar conditions will be difficult to achieve and turbulent flows are to be expected. The final nanoparticles are not only affected by the evolution of particle size and morphology through coagulation, and sintering but also through the impact of turbulence on the local particle number concentration [1]. For research on these complicated processes, the SpraySyn burner was developed to allow the experimental and numerical efficient study of spray flame synthesis of nanoparticles within the DFG (German Research Foundation) priority program SPP1980 [2]. Numerical studies of nanoparticle synthesis in turbulent flames are limited. Weise et al. [3] and Rittler et al. [4] investigated the lab-scale spray flame burner (Tethis S.p.A.) that consists of a spray nozzle and a pilot flame similar to SpraySyn burner. The former study used the Reynolds-averaged Navier-Stokes (RANS) technique, and the latter study used large eddy simulation (LES) to describe the turbulent flow. In both of these studies, the spray was modeled by Lagrangian droplet particles, and the nanoparticle growth through coagulation and coalescence were estimated using a monodisperse model $[5,6]$. The combination of the LES approach and the monodisperse model showed its feasibility in the previous study [4] and is applied to the SpraySyn burner in the present work.

\section{SpraySyn burner}

The SpraySyn burner consists of a thin tube $\left(D_{\text {in } / \text { out }}=0.4 / 0.7 \mathrm{~mm}\right)$ for the precursor solution surrounded by a nozzle for dispersion gas $(D=1.5 \mathrm{~mm})$ and followed by a wide sinter matrix 
$(D=70 \mathrm{~mm})$ providing hot pilot flame products and sheath gas. From the thin tube, a solution of $0.05 \mathrm{ml}$ iron(III)-nitrate nonahydrate per liter of Ethanol is injected with a volume flow rate $2 \mathrm{ml} / \mathrm{min}$ at ambient pressure. The whole process can be divided into three steps: a) the evaporation process of the precursor-solution mixture in the shear layer between dispersion gas and hot pilot flame, b) the turbulent combustion from the evaporated fuel and oxidizer mixture, and c) the formation and growth of $\mathrm{Fe}_{2} \mathrm{O}_{3}$ nanoparticles as schematically shown in Fig. 1.

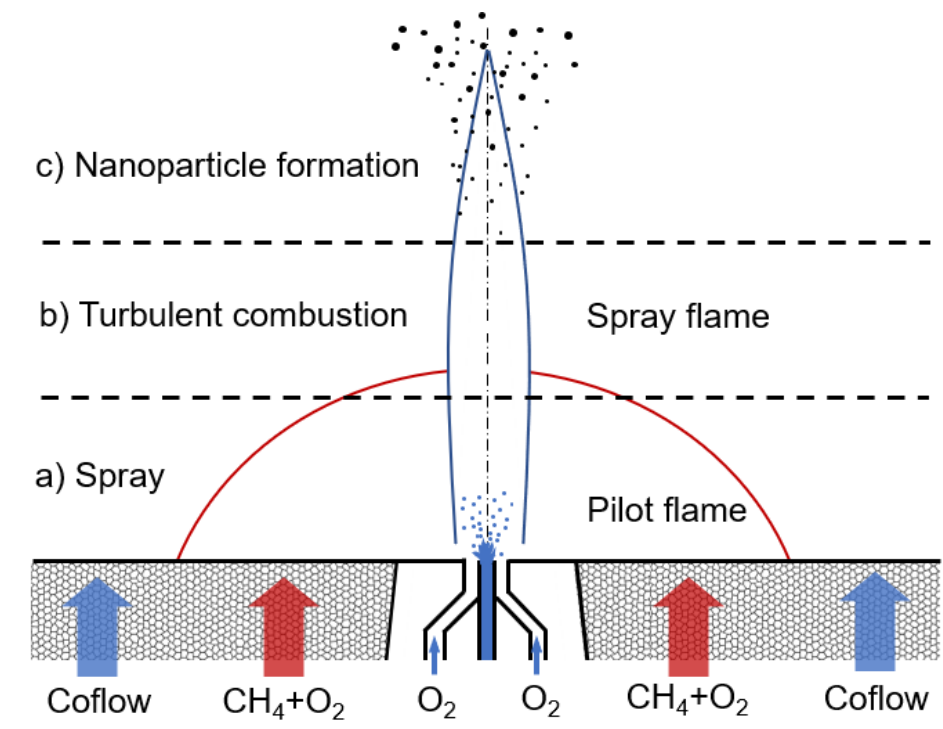

Figure 1. A schematic drawing of the burner inlet and the main processes.

Table 1. Geometric features of the inflow parameters used in simulations.

\begin{tabular}{l|ccc}
\hline & $\begin{array}{c}\text { Inner radius } \\
\mathrm{mm}\end{array}$ & $\begin{array}{c}\text { Outer radius } \\
\mathrm{mm}\end{array}$ & $\begin{array}{c}\text { Inlet velocity } \\
\mathrm{m} / \mathrm{s}\end{array}$ \\
\hline Dispersion & 0.0 & 0.75 & 103.58 \\
Dispersion (finer grid) & 0.35 & 0.75 & 132.43 \\
Pilot & 3.0 & 13.5 & 4.77 \\
Coflow & 13.5 & 35.0 & 0.67
\end{tabular}

\section{Modeling approach}

\section{Turbulent flow field modeling}

In the present study, LES is used to solve the Favre-filtered Navier-Stokes equation in a lowMach condition as:

$$
\begin{aligned}
& \frac{\partial \bar{\rho}}{\partial t}+\frac{\partial \bar{\rho} \tilde{u}_{i}}{\partial x_{i}}=\dot{\Gamma}_{\bar{\rho}} \\
& \frac{\partial \bar{\rho} \tilde{u}_{i}}{\partial t}+\frac{\partial \bar{\rho} \tilde{u}_{i} \tilde{u}_{j}}{\partial x_{j}}=-\frac{\partial \bar{p}}{\partial x_{i}}+\frac{\partial \bar{\tau}_{i j}}{\partial x_{j}}+\frac{\partial \tau_{i j}^{s g s}}{\partial x_{j}}+\dot{M}_{d, i}
\end{aligned}
$$

The equations contain the filtered density and the Favre-filtered quantities $(\bar{\rho} \tilde{\phi})$. The quantities are the pressure $p$, the stress tensor $\tau_{i j}$, and the mass $\dot{\Gamma}_{\bar{\rho}}$ and $\dot{M}_{d, i}$ momentum source terms for interaction with the liquid phase. With the filter, large eddies are resolved directly, while small eddies are modeled by Nicoud's sigma model [7]. 


\section{Spray modeling}

Since it is assumed that the primary and secondary breakup process of spray is finished close the burner surface already, the liquid phase is modeled using Lagrangian particles that represent physical liquid droplets. These numerical liquid particles are characterized by position $x_{d, i}$, velocity $u_{d, i}$, mass $m_{d}$ and temperature $T_{d}$ determined from the following differential equations with equilibrium conditions $[8,9]$ as:

$$
\begin{aligned}
\frac{d x_{d, i}}{d t} & =u_{d, i} \\
\frac{d u_{d, i}}{d t} & =\frac{f_{1}}{\tau_{d}}\left(\tilde{u}_{i}-u_{d, i}\right)+\left(1-\frac{\bar{\rho}}{\rho_{d}}\right) g_{i} \\
\frac{d m_{d}}{d t} & =-\frac{\operatorname{Sh} m_{d}}{3 \mathrm{Sc} \tau_{d}} \ln \left(1+B_{m}\right) \\
\frac{d T_{d}}{d t} & =\frac{\operatorname{Nu} c_{p}}{3 \operatorname{Pr} c_{p, d}} \frac{T-T_{d}}{\tau_{d}} \frac{\ln \left(1+B_{h}\right)}{B_{h}}+\frac{\dot{m}_{d} L_{d}}{m_{d} c_{p, d}}
\end{aligned}
$$

Where $g_{i}, T, T_{d}, c_{p}, c_{p, d}$, and $L_{d}$ denote the the gravitational acceleration, the gas and droplet temperature, the gas and droplet heat capacity, and the latent heat of vaporization, respectively. The droplet relaxation time $\tau_{d}=\left(\rho_{d} / d_{d}^{2}\right) /(18 \mu)$ is determined from the droplet diameter $d_{d}$ and effective gas viscosity $\mu$. The drag coefficient $f_{1}$ is defined with the droplet Reynolds number $R e_{d}=\left(\rho\left|\tilde{u}_{i}-u_{d, i}\right| d_{d}\right) /\left(\mu_{d}\right)$ as $f_{1}=1+0.15 R e_{d}^{0.687}$. The Schmidt Sc, Sherwood Sh, Nusselt $\mathrm{Nu}$, Prandtl Pr numbers, and the Spalding number for mass transfer $B_{m}$ and for heat transfer $B_{h}$ are used to determine the change of the droplet mass and temperature.

The publications by Rittler et al. [4, 10] can be consulted for a further discussion and implementation of the liquid phase modeling.

\section{Combustion modeling}

The gas-phase combustion is represented using the Premixed Flamelet Generated Manifold (PFGM) approach [11, 12]. In this approach, one-dimensional freely propagating laminar flames (flamelets) are computed with the Cantera library [13] using a target reaction mechanism. The chemical solutions of these flamelets are tabulated into a manifold, where the compositions and progress spaces are controlled by two mixture fractions of the burned pilot products $\left(Z_{1}=Y_{\mathrm{CO}_{2}}+Y_{\mathrm{H}_{2} \mathrm{O}}+Y_{\mathrm{O}_{2}}+Y_{C O}\right)$ and the (unburned) evaporated spray $\left(Z_{2}=Y_{E t O H}+Y_{\text {prec }}\right)$, and the progress variable $\left(Y_{p}=Y_{C O}+Y_{C O 2}\right)$. These $Z_{1}, Z_{2}$ and $Y_{p}$ are transported during the LES to retrieve the thermochemical state [14] with the Artificially Thickened Flame (ATF) approach via the efficiency function $E$ and thickening factor $F$ [15] as:

$$
\begin{aligned}
& \frac{\partial \bar{\rho} \tilde{Z}_{\alpha}}{\partial t}+\frac{\partial \bar{\rho} \tilde{u}_{i} \tilde{Z}_{\alpha}}{\partial x_{i}}=\frac{\partial}{\partial x_{i}}\left(\bar{\rho} D_{\mathrm{eff}} \frac{\partial \tilde{Z}_{\alpha}}{\partial x_{i}}\right)+\dot{\Gamma}_{\tilde{Z}_{\alpha}} \\
& \frac{\partial \bar{\rho} \tilde{Y}_{p}}{\partial t}+\frac{\partial \bar{\rho} \tilde{u}_{i} \tilde{Y}_{p}}{\partial x_{i}}=\frac{\partial}{\partial x_{i}}\left(\left[F E D_{p}+(1-\Omega) \frac{\mu_{t}}{\mathrm{Sc}_{t}}\right] \frac{\partial \tilde{Y}_{p}}{\partial x_{i}}\right)+\frac{E}{F} \dot{\omega}_{p}+\dot{\omega}_{p, e v p}
\end{aligned}
$$

The effective diffusivity is defined as sum of molecular and turbulent diffusivity $D_{\text {eff }}=D+D_{t}$, where $D_{t}$ is estimated from the turbulent viscosity $\mu_{t}$ and turbulent Schmit number $S c_{t}$ as $D_{t}=$ $\mu_{t} / \mathrm{Sc}_{t}$. The mixture fraction source term $\dot{\Gamma}_{\tilde{Z}_{\alpha}}$ represents phase exchange due to evaporation. In Eq. (8), $D_{p}, \Omega, \dot{\omega}_{p}$, and $\dot{\omega}_{p \text {,evp }}$ are the diffusivity of progress variable, flame sensor [16], and chemical source term due to the chemical reaction and evaporation, respectively.

In the present study, a combined reaction mechanism for the iron compounds $[17,18]$ is precomputed together with a reduced mechanism for ethanol originating from Olm et al. [19]. The final reaction scheme consists of 79 species and 251 reactions. 

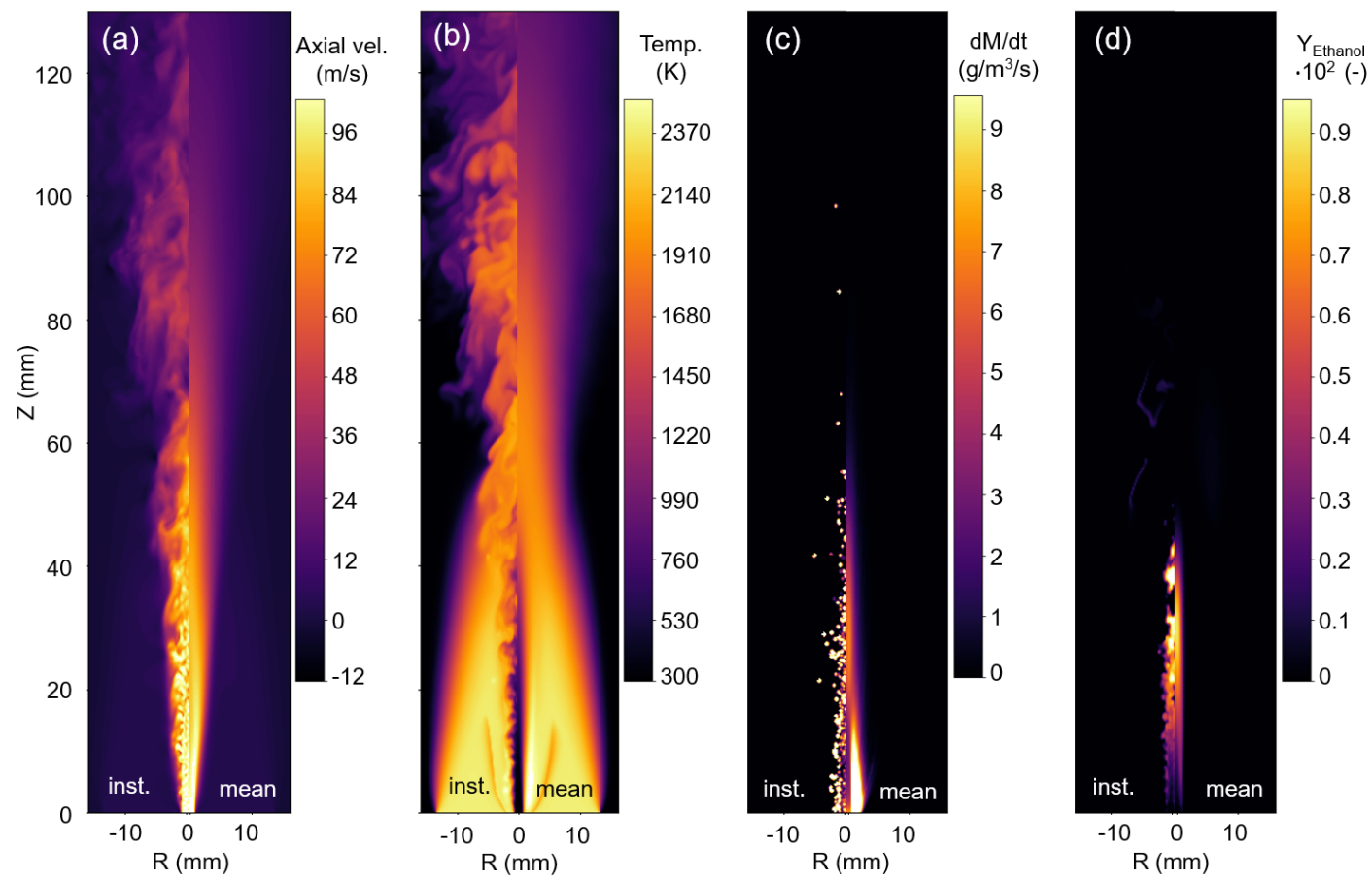

Figure 2. Instantaneous (left) and mean (right) contour plots of (a) the gas phase velocity $u$, (b) temperature, (c) evaporated precursor/solvent $d M$, and (d) mass fraction of Ethanol $Y_{\text {Ethanol }}$ in a burner cross section.

\section{Nanoparticle modeling}

The formation and growth of Iron oxide nanoparticles are estimated by the monodisperse model $[5,6]$ which contains the Favre filtered transport equations of the number-, surface-, and volume-concentration as:

$$
\begin{aligned}
\frac{\partial \bar{\rho} \widetilde{N}}{\partial t}+\frac{\partial \bar{\rho}}{\partial x_{j}}\left(\widetilde{N} \bar{u}_{j}-D_{\text {eff }} \frac{\partial \widetilde{N}}{\partial x_{j}}\right) & =-\frac{1}{2} \beta \bar{\rho} \widetilde{N}^{2}+\bar{\rho} \widetilde{I}, \\
\frac{\partial \bar{\rho} \widetilde{A}}{\partial t}+\frac{\partial \bar{\rho}}{\partial x_{j}}\left(\widetilde{A} \bar{u}_{j}-D_{\text {eff }} \frac{\partial \bar{A}}{\partial x_{j}}\right) & =-\frac{1}{\tau} \bar{\rho}\left(\widetilde{A}-\widetilde{N} a_{s}\right)+\bar{\rho} \widetilde{I} a_{m}, \\
\frac{\partial \bar{\rho} \widetilde{V}}{\partial t}+\frac{\partial \bar{\rho}}{\partial x_{j}}\left(\widetilde{V} \widetilde{u}_{j}-D_{\text {eff }} \frac{\partial \widetilde{V}}{\partial x_{j}}\right) & =\bar{\rho} \widetilde{I} v_{m} .
\end{aligned}
$$

Where $\beta, \tau, a_{s}, a_{m}$ and $v_{m}$ are the coagulation kernels, the specific sintering time, the surface area for the completely fused particles, and the surface area of the monomer. The nucleation source term I represents the newly produced monomer particles and it is precomputed and tabulated with other gas-phase components. The $\mathrm{Fe}_{2} \mathrm{O}_{3}$ monomer diameter is assumed as $0.45 \mathrm{~nm}$, and the diameter of aggregate particle is computed with $d_{a}=(6 \mathrm{~V} /(N \pi))^{1 / 3}$. For further information of the monodisperse model with the LES concept, the paper by Rittler et al. [4] should be consulted.

\section{Numerical setup}

The simulations were conducted with the LES in-house solver PsiPhi [10, 16, 20], which was applied and validated in various LES and DNS studies. The inflow turbulence was generated by the method developed by Klein et al. [21] in an efficient implementation [22].

The main simulations with nanoparticle synthesis were performed in a domain $135 \times 35 \times 35 \mathrm{~mm}^{3}$

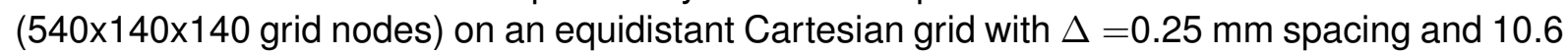
million cells. Since the spray nozzle located in the burner center has a $0.75 \mathrm{~mm}$ diameter, the computational domain starts $3 \mathrm{~mm}$ from the burner surface to circumvent the necessity for a 


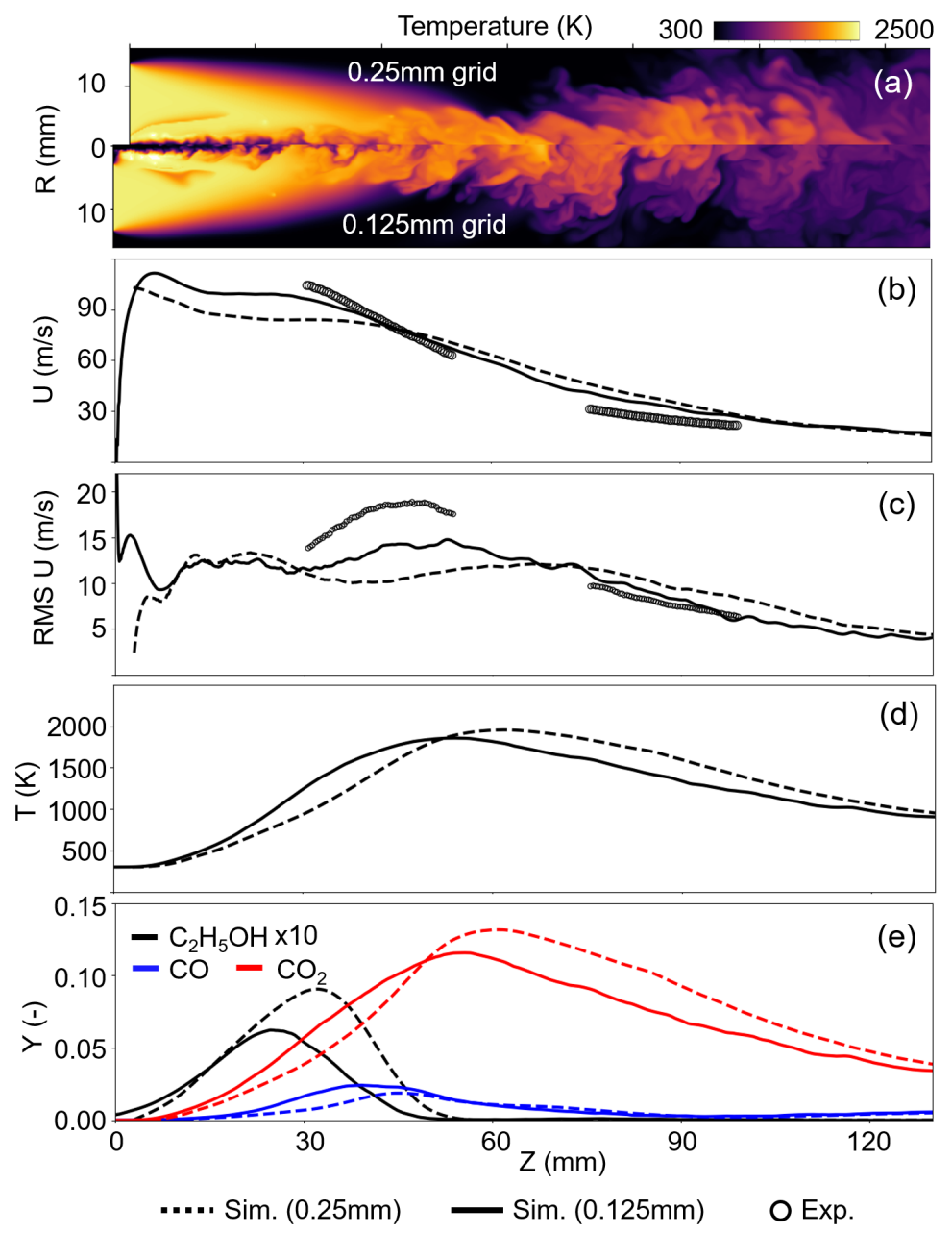

Figure 3. Instantaneous contour plots of (a) temperature with the $0.25 \mathrm{~mm}$ grid (top) and 0.125 grid (bottom). Axial centerline profiles of the (b) mean-, (c) RMS axial velocity, (d) temperature, and (e) mass fraction of major species.

finer grid size. An additional simulation without nanoparticle model was performed with a larger domain $150 \times 45 \times 45 \mathrm{~mm}^{3}$ with $\Delta=0.125 \mathrm{~mm}$ (155 million cells) from the burner surface to test the grid size effect. Further details of the simulation inflow setup are summarised in Table 1.

The costs of simulations for 0.2 seconds are 26,000 core hours for the main simulation and 172,000 core hours for the finer grid respectively.

Setting spray boundary conditions requires to seed droplets at the inlet plane such that they satisfy the correct joint distribution or probability density function (PDF) $p(r, u, v, D)$, for the radial coordinate $r$ the axial and radial droplet velocities $u, v$ and the droplet diameter $D$. Such a PDF is available from the experiments, and suitable spray droplets for the simulation could be generated from it. However, this is prone to error, costly, furthermore, a suitable way would need to be found for describing the PDF from the experiments. Instead, we use the droplet data directly, seeding droplets with tuples of measured data [23]. In order to preserve the proper liquid mass flow, the seeded mass is monitored and occurring misbalance, caused by the discrete nature of the process, is corrected over the next time steps.

\section{Results and discussion}

Figure 2 shows contour plots of predicted instantaneous (left) and mean (right) axial velocity, temperature, evaporated precursor/solvent mass source, and mass fraction of ethanol in the burner cross-section. The zone from the nozzle exit to $50 \mathrm{~mm}$ axial distance is denoted as the upstream region. In this region, mixing occurs between the hot pilot flame and the dispersion 


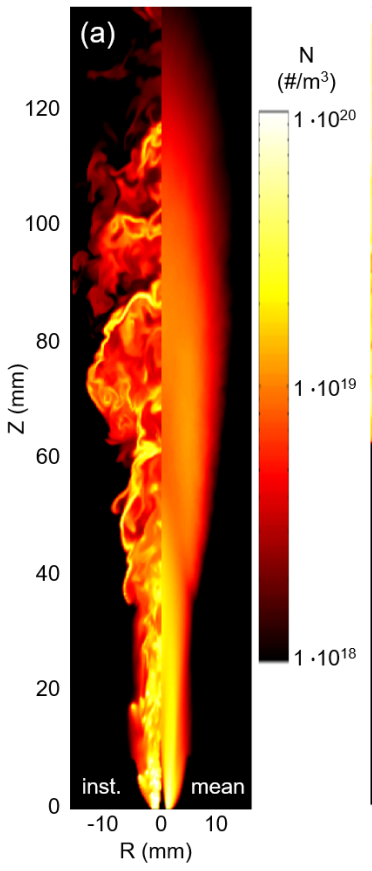

(b)

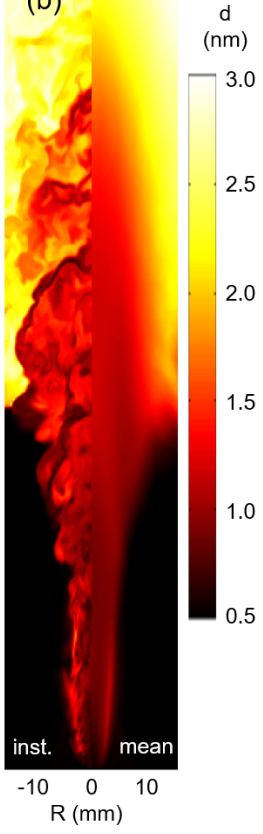

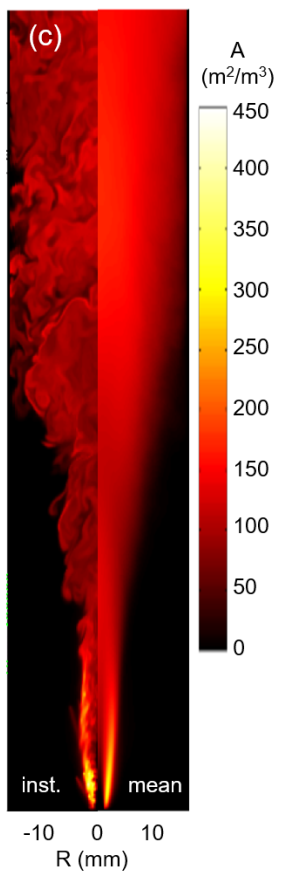

Figure 4. Instantaneous (left) and mean (right) contour plots of (a) the particle number concentration $N$, (b) aggregate particle diameter $d$, and (c) surface area concentration $A$ in a burner cross section.

gas containing liquid droplets. Turbulent structures are found in the shear layer between fast dispersion gas and the co-flow. These shear layers produced by momentum differences lead the heat penetrating to increase. The liquid droplets are evaporated in a narrow, long region up to $50 \mathrm{~mm}$ above the burner. The averaged evaporation rate $d \dot{M}$ shows that most of the evaporation happens near the inlet.

Figure 3 shows differences of predicted axial velocity, temperature and major species between the simulation with $0.25 \mathrm{~mm}$ grid and $0.125 \mathrm{~mm}$ grid along the center axis. As shown in Fig. $3(b, c)$ predicted axial velocity of both simulation have a good agreement with the experiment [24], and (d,e) show that the peak location of temperature and mass fraction of major species are affected by the inclusion of the near the burner surface as computational domain.

Figure $4(\mathrm{a}, \mathrm{b}, \mathrm{c})$ shows contour plots of predicted particle number concentration $N$, aggregate particle diameter $d$, surface area concentration $A$ in a burner cross-section. In the diameter plot, larger particles can be observed at the edges than in the centre. This is consistent with the plot of number concentration and surface concentration. The velocities at the edges are lower, which is associated with longer residence times. The particles have more time to coagulate and the number concentration decreases, while particles are still visible in the surface plot. The surface area concentration increases strongly in the shear layer between dispersion and hot pilot flame due to the nucleation of monomers shown in Fig. 4 (c).

Figure 5 shows the particle number concentration and aggregate particle diameter along the centerline. The particle number concentration increases with the formation of $\mathrm{Fe}_{2} \mathrm{O}_{3}$ monomers and decreases afterwards due to the coagulation in the high-temperature region and dilution further downstream by the sheath gas as shown in Fig. 4 (a) and Fig. 5. The profile of the aggregated particle diameter estimated by $V$ and $N$ allows observing the growth of particles with the height of the burner. The aggregate particle diameter is increased due to the coagulation process.

\section{Conclusions}

In the present work, a large eddy simulation (LES) of nanoparticle synthesis with SpraySyn burner has been performed. The cost-effective monodisperse model is combined with the LES 


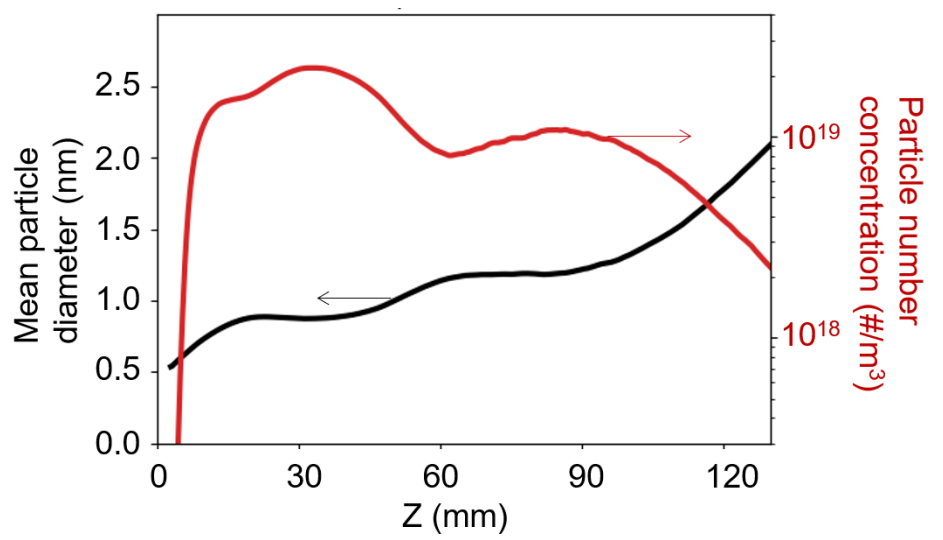

Figure 5. Axial centerline profiles of the aggregate particle diameter $d$ (black) and the particle number concentration $N$ (red).

approach to investigate $\mathrm{Fe}_{2} \mathrm{O}_{3}$ nanoparticle formation in a spray flame, and showed its ability to predict nanoparticle generation at an appropriate computational cost.

Iron-nitrate precursor dissolved in Ethanol solvent is evaporated mainly in the shear layer between the hot pilot flame and dispersion gas. The primary $\mathrm{Fe}_{2} \mathrm{O}_{3}$ monomers source is precalculated and tabulated with other gas-phase reactions in a premixed flamelet generated manifolds (PFGM) approach.

\section{Acknowledgements}

The authors gratefully acknowledge the financial support from the German Research Foundation (DFG, grant number KE-1751-11-1) and the use of the "Gauss Centre for Supercomputing (GCS)," the "Center for Computational Sciences and Simulation" for computing time on the GCS Supercomputer SuperMUC-NG and MagnitUDE (DFG, grant Number INST 20876/209-1 FUGG and INST 20876/243-1 FUGG).

\section{References}

[1] Cifuentes, L., Sellmann, J., Wlokas, I., and Kempf, A., 2020. "Direct numerical simulations of nanoparticle formation in premixed and non-premixed flame-vortex interactions". Physics of Fluids, 32(9), p. 093605.

[2] Schneider, F., Suleiman, S., Menser, J., Borukhovich, E., Wlokas, I., Kempf, A., Wiggers, H., and Schulz, C., 2019. "Spraysyn- a standardized burner configuration for nanoparticle synthesis in spray flames". Review of Scientific Instruments, 90, 08, p. 085108.

[3] Weise, C., Menser, J., Kaiser, S., Kempf, A., and Wlokas, I., 2015. "Numerical investigation of the process steps in a spray flame reactor for nanoparticle synthesis". Proceedings of the Combustion Institute, 35(2), pp. 2259-2266.

[4] Rittler, A., Deng, L., Wlokas, I., and Kempf, A. M., 2016. "Large eddy simulations of nanoparticle synthesis from flame spray pyrolysis". Proc. Combust. Inst., 36, pp. 1077 1087.

[5] Kruis, F. E., Kusters, K. A., Pratsinis, S. E., and Scarlett, B., 1993. "A simple model for the evolution of the characteristics of aggregate particles undergoing coagulation and sintering". Aeros. Sci. Technol., 19(4), pp. 514-526.

[6] Panda, S., and Pratsinis, S. E., 1995. "Modeling the synthesis of aluminum particles by evaporation-condensation in an aerosol flow reactor". Nanostructured Materials, 5(7-8), pp. 755-767.

[7] Nicoud, F., Toda, H. B., Cabrit, O., Bose, S., and Lee, J., 2011. "Using singular values to build a subgrid-scale model for large eddy simulations". Physics of Fluids, 23(8), 
p. 085106.

[8] Abramzon, B., and Sirignano, W. A., 1989. "Droplet vaporization model for spray combustion calculations". International Journal of Heat and Mass Transfer, 32(9), pp. 1605-1618.

[9] Miller, R. S., Harstad, K., and Bellan, J., 1998. "Evaluation of equilibrium and nonequilibrium evaporation models for many-droplet gas-liquid flow simulations". International Journal of Multiphase Flow, 24(6), pp. 1025-1055.

[10] Rittler, A., Proch, F., and Kempf, A. M., 2015. " $\{L E S\}$ of the sydney piloted spray flame series with the $\{$ PFGM $\} /\{$ ATF $\}$ approach and different sub-filter models". Combust. Flame, 162(4), pp. 1575 - 1598.

[11] van Oijen, J. A., and de Goey, L. P. H., 2000. "Modelling of premixed laminar flames using flamelet-generated manifolds". Combust. Sci. Technol., 161(1), pp. 113-137.

[12] van Oijen, J. A., Lammers, F. A., and de Goey, L. P. H., 2001. "Modeling of complex premixed burner systems by using flamelet-generated manifolds". Combust. Flame, 127(3), pp. $2124-2134$.

[13] Goodwin, D. G., Speth, R. L., Moffat, H. K., and Weber, B. W., 2018. Cantera: An objectoriented software toolkit for chemical kinetics, thermodynamics, and transport processes. https://www . cantera. org. Version 2.4.0.

[14] Peters, N., 2000. Turbulent Combustion. Cambridge University Press.

[15] Kuenne, G., Ketelheun, A., and Janicka, J., 2011. "Les modeling of premixed combustion using a thickened flame approach coupled with fgm tabulated chemistry". Combust. Flame, 158(9), pp. $1750-1767$.

[16] Proch, F., and Kempf, A. M., 2014. "Numerical analysis of the cambridge stratified flame series using artificial thickened flame les with tabulated premixed flame chemistry". Combustion and Flame, 161(10), pp. 2627-2646.

[17] Feroughi, O. M., Hardt, S., Wlokas, I., Hülser, T., Wiggers, H., Dreier, T., and Schulz, C., 2015. "Laser-based in situ measurement and simulation of gas-phase temperature and iron atom concentration in a pilot-plant nanoparticle synthesis reactor". Proceedings of the Combustion Institute, 35(2), pp. 2299-2306.

[18] Oh, J., and Noh, D., 2017. "The reduction kinetics of hematite particles in h2 and co atmospheres". Fuel, 196, pp. 144-153.

[19] Olm, C., Varga, T., Valkó, É., Hartl, S., Hasse, C., and Turányi, T., 2016. "Development of an ethanol combustion mechanism based on a hierarchical optimization approach". International Journal of Chemical Kinetics, 48(8), pp. 423-441.

[20] Proch, F., Domingo, P., Vervisch, L., and Kempf, A. M., 2017. "Flame resolved simulation of a turbulent premixed bluff-body burner experiment. Part I: Analysis of the reaction zone dynamics with tabulated chemistry". Combust. Flame, 180, pp. 321-339.

[21] Klein, M., Sadiki, A., and Janicka, J., 2003. "A digital filter based generation of inflow data for spatially developing direct numerical or large eddy simulations". Journal of Computational Physics, 186(2), pp. 652-665.

[22] Kempf, A. M., Wysocki, S., and Pettit, M., 2012. "An efficient, parallel low-storage implementation of klein's turbulence generator for les and dns". Computers \& Fluids, 60, pp. 58-60.

[23] Schneider, F., Dreier, T., and Schulz, C., 2019. "SpraySyn - standard burner for the collaborative investigation of spray-flame synthesis of nanoparticles: Droplet velocity and size of the non-reactive ethanol spray". European Combustion Meeting, Lisbon, Portugal, 9.

[24] Martins, F. J., Kirchmann, J., Kronenburg, A., and Beyrau, F., 2020. "Experimental investigation of axisymmetric, turbulent, annular jets discharged through the nozzle of the spp1980 spraysyn burner under isothermal and reacting conditions". Experimental Thermal and Fluid Science, 114, p. 110052. 